\title{
Research Progress of Microbial Analyzing Methods in the Wetland Ecosystem
}

\author{
Zhenzhen Wan ${ }^{1, a}$, Qin Cheng ${ }^{2, b}$, Wei Pan ${ }^{2, c}$ and Haijun Liü, ${ }^{2}$ \\ ${ }^{1}$ Department of Postgraduate Education, Anqing Normal University, 1318 Jixian North Road, \\ Anqing 246133, P.R. China \\ ${ }^{2}$ School of Resources and Environment, Anqing Normal University, 1318 Jixian North Road, Anqing \\ 246133, P.R. China \\ ªzzscut@163.com, ${ }^{\mathrm{b}} 15665335700 @ 163 . c o m,{ }^{c} 1993$ panwei@163.com, ${ }^{\mathrm{d}^{*}}$ navyctgu@163.com
}

Keywords: Microorganism; Wetland ecosystem; Molecular biological method; Research progress Abstract: As one of the most important components of the wetland ecosystem, microorganisms play an important role in all kinds of ecological processes. Those ecological processes of wetland microorganisms are mainly represented by elements cycling, material transformation, energy flow and ecological restoration. In this paper, the research progress of microbial analyzing methods in the wetland ecosystem are introduced clearly and have shown their specific advantages and disadvantages. They mainly include traditional microorganism counting methods, microbial physiological biochemical index methods and molecular biological techniques. This paper will help us better understand the research progress of wetland microorganisms.

\section{Introduction}

Microorganisms play an important role in elements cycling and material transformation in wetland ecosystem. They are the most important decomposers in ecosystem that help the ecosystem recover small molecules and soil enzymes. Microbial secretions and those elements from microorganism death could convert to the nutrient substances for other organisms in environmental ecosystem directly or indirectly. Such as, microorganisms are the significance driving forces of the biogeochemical cycles like nitrogen and phosphorus elements. Furthermore, microorganisms can accelerate the decomposition progress of organisms and transform them into small molecules to be used by other organisms in ecosystem. Microorganisms not only decompose various biological metabolites and dead body into simple inorganic matter, but also degrade various synthetic compounds and speed up the elements cycling. Obove all, microorganisms are taking an important part in the wetland ecosystem, and how to analyze microbial communities, structures and functions have became a popular issues.

\section{Research methods of wetland microorganisms}

The research methods of wetland microorganisms mainly includes the traditional technologies technologies such as all kinds of microscopical technologies, and the pure culturable technologies, as well as the modern molecular technologeis.

\section{Traditional microbial research analysis}

The traditional microbial research methods include microscopical technologies, aseptic technologies and pure culturable technologies. The microscopical technology could magnify microorganisms 
several times that be seen by naked eyes and open the door of the microscopic world for people. The aseptic technology constructs a foundation for the pure culturable technology and the molecular technology. Pure culturable technology realizes the laboratory cultivation and observation of microorganism units and microbial communities. Although the microscopical technology and aseptic technology could be used for counting the number of microorganisms in wetland ecosystem, but those two methods might obtain different results. Because microscopical counting was counted directly in the sight, while the pure culturable technology was indirectly estimating the number of microorganisms by microbial community in the culturable mediums. In addition to, the pure culturable technology could lead to the microbial enrichment growth of some special species. Meanwhile, not all microorganisms could be cultured under the current conditions that makes the results of those two methods are different. Although there is a limit in the pure culturable technology, but it doesn't affect its status for studying microorganisms in the wetland ecosystem. What's more, some improved methods such as microscopic counting with dyes, mixed bacteria methods and the most probable number (MPN) to count microorganisms are developed based on the the basic microscopical technology and the pure culturable technology, which has been widely used in the microbial research in the wetland ecosystem.

\section{Microbial physiological-biochemical indexs analysis}

Recently, those researchers pay more attention to microbiological targets that could reflect microbial activity and wetland health like soil biomass, soil enzyme and the carbon substrate metabolic ability. The microbial biomass usually is the potential material source of nutrients for other organisms in wetland ecosystem. Soil respiration can represent the activity of soil microorganisms in some degree, while soil enzyme is the result of the activity to soil microorganisms, animals and plants in ecosystem. The outside measurment of the microbial biomass mainly is in situ observation, while there are more measurment methods for indoor microbial biomass such as the chloroform fumigation-sodium bicarbonate extraction method, the chloroform fumigation- $\mathrm{K}_{2} \mathrm{SO}_{4}$ extraction method, as well as the carbon isotopic tracing. The measurment of soil respiration not only are achieved outside, but also obtained using indoor determination of alkali absorption. Now, they are popular for soil respiration by the gas chromatography and $\mathrm{CO}_{2}$ infrared analysis methods. Meanwhile, the study of soil microbial enzyme activity mainly focuses on the correlation of the enzyme activity and soil microorganisms. The analyzing methods of soil enzyme mainly include the traditional titration methods, as well as the high performance liquid chromatography (HPLC).

Recently, those researches on microbial communities in wetland ecosystem have developed since the introduction of the technology based on fatty acids of biological membranes. For instance, the Phospholipid fatty acid (PLFA) analysis and Fattyacid methyl esters (FAMEs) analysis are depended on the composition and abundance of the different phospholipid fatty acid in microbial membranes, while those species have their own characteristics. Therefore it can be an uesful biomarker for different kinds of microorganisms in the wetland ecosystem. As saturated fatty acid often take as the symbol for the total biomass of microbial community in the ecosystem. The microbial community structure in wetland ecosystem might be represented clearly by analyzing the biomarker. And the data of PLEA and FAMEs were obtained on the individual databases based on living cells in wetland ecosystem. Meanwhile, the biology system was built by Garland and Miss to study the microbial community structure and microbial functions of soil microorganism, while was first applied to identify the pure microorganisms in environmental ecosystem. Then, it was improved and introduced to the research of microbial community metabolic functions. 


\section{Molecular biological analysis}

Those culturable microorganisms is limited in the environment that usually could not show the real condition of microbial community. Traditional microbial research methods are commonly based on microscopical technologies, aseptic technologies and pure culturable technologies which just show the general description of microbial characteristics in the environment. It is necessary to improve the present microbial research methods for better understanding the characteristics of soil microbial community, structure and their functions. Thus, polymerase chain reaction (PCR) technology and those molecular technologies based on PCR can conduct researches and analyze microorganisms in soil environment and also don't need to rely on the pure culture, which create a new researching situation for soil microorganisms.

Those molecular technologies that applied to wetland microbial researches can be divided into two categories. First kind is DNA probe and genechip that based on molecular hybridization. DNA probe and genechip could acquire the genetic information of unknown fragments by using the hybridization signal with the known relative probes and DNA sequences. The other kind based on PCR technology are widely used. Through PCR can get more interesting fragments like the functional gene fragments nirK and nirS of nitrite reductase, which is good for the further assay determination of purpose fragments. There are various of microbial research methods based on the PCR technology, such as denaturing gradient gel electrophoresis (DGGE), temperature gradient gel electrophoresis (TGGE), terminal restriction fragment length polymorphism (T-RFLP), single strand conformation polymorphism (SSCP), random amplified polymorphic DNA (RAPD) and length heterogeneity PCR (LH-PCR). Those molecular biological methods help us better understand the unknown microorganism world.

\section{Conclusion}

Soil microbial researches gradually become a hot topic on the wetland system. And the continuous improvement and innovation of the traditional microbial research methods, especially the application of molecular biologcial techniques creates a new situation in the microbial research in wetland ecosystem. But each technique has its own advantages, disadvantages and its emphasis. The traditional microbiological methods could only show the general description on the specific microbial phenotypic characteristics, furthermore, there is possibility that underestimates microbial community diversity. Compared with the generality of those traditional analyzing methods, modern molecular technologies could be widely applied to analyze most microorganisms, not only the culturable microbes, but also the unculturable microbes. However, the accuracy is usually affected by many factors. Therefore, the research on wetland microorganisms need conduct the effective combination of a variety of methods, especially the combination of traditional methods and molecular technologies, such as microscopical technologies, molecular biological methods, Metagenomics and the isotopic tracing technology, which will show a better interpretation of microbial communities, structures and functions in wetland ecosystem.

\section{Acknowledgements}

This work was supported by grants from National Natural Science Foundation of China (41473096), the Doctoral Scientific Research Funds of Anqing Normal University (150002000007). 


\section{References}

[1] Chauhan A OA, Reddy KR. Syntrophic methanogenic associations along a nutrient gradient in Florida Everglades. Appl Environ Microbiol. 2004;70:3475-3484.

[2] Benner R MA, Hodson RE. Anaerobic biodegradation of the lignin and polysaccharide components of lignocelluloses and synthetic lignin by sediment micro flora. Appl Environ Microbiol. 2009;47:998-1004.

[3] Stout LM NK. Shifts in rhizoplane communities of aquatic plantsafter cadmium exposure. Appl Environ Microbiol. 2006;71:2484-2492.

[4] Brookes PC, Polson DS, Jenkinson DS. Measurement of microbial biomass phosphorus in soil. Soil Boil Brioche. 2010;380:163-172.

[5]Busing N, Gessner MO. Benthic bacterial and fungal productivity andcarbon turnover in a freshwater marsh. Appl Environ Microbiol,2010;67:308-319.

[6] S. Yousaf, V. Andria, T.G. Reichenauer, et al. Phylogenetic and functional diversity of alkane degrading bacteria associated with Italian ryegrass (Lolium multiflorum) and Birdsfoot trefoil (Lotus corniculatus) in a petroleum oil-contaminated environment[J]. J Hazard Mater, 2010; 184: 523-532. [7] P. Bombach, N. Nägele, M. Rosell, et al. Evaluation of ethyl tert-butyl ether biodegradation in a contaminated aquifer by compound-specific isotope analysis and in situ microcosms[J]. J Hazard Mater, 2015;286: 100-106.

[8] D. Dunn. Ammonium tolerance and toxicity of Actinoscirpus grossus-A candidate species for use in tropical constructed wetland systems [J]. Ecotox Environ Safety, 2014; 107: 329-329. 